\title{
Sensory stimulation in the treatment of children with sleep-related rhythmic movement disorder: a feasibility and acceptability study
}

Rachel M. van Sluijs ${ }^{1,2^{*}}$ D, Elisabeth Wilhelm¹, Quincy J. Rondei ${ }^{1}$, Lukas Jäger ${ }^{1}$, Markus Gall ${ }^{3}$, Heinrich Garn ${ }^{3}$, Peter Achermann ${ }^{2,4}$, Oskar G. Jenni ${ }^{2,5}$, Robert Riener ${ }^{1,2,6}$ and Catherine M. Hill ${ }^{7,8}$

\begin{abstract}
Background: Sleep-related rhythmic movement disorder is characterized by repetitive gross-motor movements at sleep onset or during sleep, which result in clinical consequences such as impact on daytime functioning and injury. No well-established therapies exist today. Substituting the patient's movements with external sensory stimulation may offer a treatment modality. The aim of the current study was to test the feasibility and acceptability of vestibular stimulation using a rocking bed (Somnomat) in children with rhythmic movement disorder and to assess children's movement preference.

Methods: Children with rhythmic movement disorder ( $n=6$, Age: 5-14 years) were studied over three nights in a sleep laboratory: adaptation night (normal bed) and randomised-order baseline (Somnomat) and intervention nights (Somnomat). Child's preferred movement direction (head-to-toe or side-to-side) and frequency (between 0.25 and $2 \mathrm{~Hz}$ ), determined during an afternoon protocol, were applied using the Somnomat for $1 \mathrm{~h}$ after lights out, and in response to subsequent episodes of rhythmic movement during intervention nights. Comfort assessed using a questionnaire, and objective sleep parameters assessed using videosomnography, were compared.

Results: The participants' sometimes violent rhythmic movements did not disturb device performance. All children rated intervention nights equally or more comfortable than baseline nights. Self-reported sleep quality, as well as the number and duration of movement episodes did not significantly differ between baseline and intervention nights.
\end{abstract}

Conclusions: Providing rocking movements using the Somnomat is both technically feasible and acceptable to the target population. The therapeutic value of this novel stimulus substitution for rhythmic movement disorder should now be evaluated in a larger sample over a longer period in the home setting.

Trial registration: The trial was retrospectively registered at clinicaltrials.gov (NCT03528096) on May 17th 2018.

Keywords: Pediatrics, Jactatio capitis nocturna, Rythmie du sommeil, Head banging, Sensory substitution, Vestibular stimulation

\footnotetext{
* Correspondence: Rachel.vansluijs@hest.ethz.ch

${ }^{1}$ Department of Health Sciences and Technology, Sensory-Motor Systems

Lab, ETH Zurich, Sonneggstrasse 3, 8092 Zurich, Switzerland

${ }^{2}$ Center of Competence Sleep \& Health Zurich, University of Zurich,

Frauenklinikstrassse 28, 8091 Zurich, Switzerland

Full list of author information is available at the end of the article
}

(c) The Author(s). 2020 Open Access This article is licensed under a Creative Commons Attribution 4.0 International License, which permits use, sharing, adaptation, distribution and reproduction in any medium or format, as long as you give appropriate credit to the original author(s) and the source, provide a link to the Creative Commons licence, and indicate if changes were made. The images or other third party material in this article are included in the article's Creative Commons licence, unless indicated otherwise in a credit line to the material. If material is not included in the article's Creative Commons licence and your intended use is not permitted by statutory regulation or exceeds the permitted use, you will need to obtain permission directly from the copyright holder. To view a copy of this licence, visit http://creativecommons.org/licenses/by/4.0/. 


\section{Background}

Sleep-related rhythmic movement disorder (RMD) is a childhood-onset sleep disorder characterized by gross motor movements occurring at sleep onset and during sleep (Manni and Terzaghi 2005) (Supplemental Information Documents 1 and 2). These nocturnal episodes of rhythmic movement may disrupt the sleep of both the sufferer and other household members with impact on daytime functioning, and can result in injury (Sateia 2014). Even though clinical consequences of sleeprelated RMD can be severe, there are no well-established therapies (Gwyther et al. 2017). While psychological, behavioural and pharmacological treatments have been reported in case studies and small observational studies (Gwyther et al. 2017), the most consistent treatment recommendations for children are based on reassurance and safety advice to reduce injury risk (Mahowald 1995; Mindell and Owens 2015; Shneerson 2009) with no quality treatment trial data to support clinical management.

Understanding what causes RMD is limited to hypotheses (Manni and Terzaghi 2005). As rhythmic sensory stimulation is known to be soothing and relaxing, (Vrugt and Pederson 1973; Grabherr et al. 2015; Omlin et al. 2016) and promotes sleep in infants and young adults, (Barnard and Bee 1983; Bayer et al. 2011; Shibagaki et al. 2017) it has been suggested that RMD might be a learned soothing, (Haywood and Hill 2012) or selfstimulating, behaviour that becomes habitual and compulsive (Clark and Chee 1977; Sallustro and Atwell 1978). At the same time, rhythmic movements might also promote early motor development (Clark and Chee 1977; Sallustro and Atwell 1978). Sallustro and colleagues found that children reported to perform daytime rhythmic movements reached motor milestones earlier, compared to peers who did not (Sallustro and Atwell 1978). Furthermore, a controlled experiment showed that infants who received experimental vestibular stimulation in a rotating chair reached motor milestones earlier than a control group (Clark and Chee 1977). While daytime rhythmic movements may have developmental advantages, when rhythmic movements disrupt sleep and result in clinical consequences this is considered a sleep disorder. Common consequences such as local injury and daytime cognitive and behavioural impairments could be minimised if the number, duration or intensity of rhythmic movements were reduced. Evaluation of therapeutic interventions for this poorly researched sleep disorder are overdue.

Sensory systems stimulated by the patients' rhythmic movements include the visual, proprioceptive, vestibular and, in the case of head banging, the auditory system (Golbin et al. 2013). Thus, one therapeutic approach could be to substitutes the sensory experience of rocking with an external source of rhythmic movement, which may down-regulate the internal drive to rock. We previously reported the benefits of vestibular stimulation, in the form of a swinging motion passively generated when sleeping in a hammock, in five children with RMD treated in the Southampton Children's Hospital sleep clinic (Hewertson and Hill 2018). Although promising, the passive movement of a hammock cannot be reliably reproduced. An automated bed that generates rocking movements offers the advantage that stimulation intensity can be controlled and manipulated (Crivelli et al. 2016). Furthermore, stimulation can be deployed at sleep onset (aiming to prevent episodes of rhythmic movement) and can be applied during the night in response to rhythmic movements.

We have developed and tested the rocking Somnomat bed in adults, but have not tested its technical capabilities with participants who may move violently in the device. The present study explored the stimulation settings that are perceived as most comfortable and likely to promote sleep by children with RMD. Furthermore, the feasibility and acceptability of rocking movements provided on demand during the night were assessed.

\section{Methods}

Recruitment

Six typically developing children with RMD (2 female) aged 5 to 14 years (Median: 8.5 years, IQR: 5.25 to 12.5 years) were included in the study (Table 1). Participants were recruited from a social media group of affected families in the UK. Eligible participants were children between 5 and 18 years of age with self-reported RMD and no self-reported sensitivity to motion sickness (Henriques et al. 2014). Diagnosis according to the International Classification of Sleep Disorders III, was confirmed by a European certified somnologist $(\mathrm{CMH})$ based on a structured interview (Supporting Information Document 3) and a home video recording (Sateia 2014; Gogo et al. 2018).

\section{Rocking bed}

Sensory stimulation was provided by the Somnomat, an actuated bed previously validated for use in healthy adults (Crivelli et al. 2016). In summary, the bed provides sinusoidal rocking movements along the trajectory of a pendulum with a centre of rotation $4 \mathrm{~m}$ above the bed. It moves in two directions: head-to-toe and side-toside (Fig. 1). For children under 10 years of age only head-to-toe direction was used for safety reasons. Pressure sensitive floor mats initiated a safety stop to prevent participants from approaching the moving mechanism. Cushioning of the bed prevented contact injuries.

The control software was adapted so that the bed frequency could be varied between 0.25 and $2.0 \mathrm{~Hz}$ at a 
Table 1 Demographics

\begin{tabular}{|c|c|c|c|c|c|c|c|c|c|}
\hline Case & Sex & Age & $\begin{array}{l}\text { Rhythmic } \\
\text { movement } \\
\text { semiology }\end{array}$ & $\begin{array}{l}\text { Habitual } \\
\text { movement } \\
\text { frequency }\end{array}$ & Injury & $\begin{array}{l}\text { Influence on } \\
\text { daytime functioning }\end{array}$ & $\begin{array}{l}\text { Influence } \\
\text { on sleep }\end{array}$ & $\begin{array}{l}\text { Onset } \\
\text { Age }\end{array}$ & $\begin{array}{l}\text { Medication } \\
\text { prior to study }\end{array}$ \\
\hline & & years & & $\mathrm{Hz}$ & & & & months & \\
\hline 1 & male & 5 & $\begin{array}{l}\text { head rolling, body } \\
\text { rolling }\end{array}$ & 1.4 & none & no & not sure & 12 & none \\
\hline 2 & male & 5 & $\begin{array}{l}\text { head banging, } \\
\text { body rocking }\end{array}$ & 1.2 & callus, bleeding & yes & a little & 9 & none \\
\hline 3 & female & 6 & $\begin{array}{l}\text { body rocking, head } \\
\text { rolling }\end{array}$ & 0.6 & $\begin{array}{l}\text { bruising, bleeding, hair } \\
\text { loss, neck pain }\end{array}$ & yes & very much & 6 & Melatonin \\
\hline 4 & male & 11 & $\begin{array}{l}\text { head banging, } \\
\text { head rolling }\end{array}$ & $2.3^{*}$ & $\begin{array}{l}\text { bruising, bleeding, hair } \\
\text { loss }\end{array}$ & no & a little & 6 & none \\
\hline 5 & female & 13 & $\begin{array}{l}\text { body rocking, body } \\
\text { rolling }\end{array}$ & 0.9 & hair loss & yes & a little & 24 & none \\
\hline 6 & male & 14 & body rocking & 0.7 & none & yes & very much & 24 & none \\
\hline
\end{tabular}

Habitual movements were determined based on video recordings and in the one child showing no movements $\left(^{*}\right)$ by voluntary demonstration of symptoms during daytime.

resolution of $0.25 \mathrm{~Hz}$. This range encompassed the full range of typical rhythmic movement frequencies (Sateia 2014). The trajectory amplitude was scaled to the frequency to achieve a maximum velocity of $0.1 \mathrm{~m} / \mathrm{s}$ resulting in amplitudes between 0.008 and $0.67 \mathrm{~m}$. As rhythmic movements can be forceful, the impact of these movements on the provided stimulation was of interest. Accuracy of the provided stimulation was assessed by calculating the root mean square error between the reference velocity and the measured velocity of the bed along its trajectory, during the first hour of the intervention for each participant.

\section{Movement preference protocol}

A strict protocol assessed participant's preference for Somnomat movement direction and intensity using a

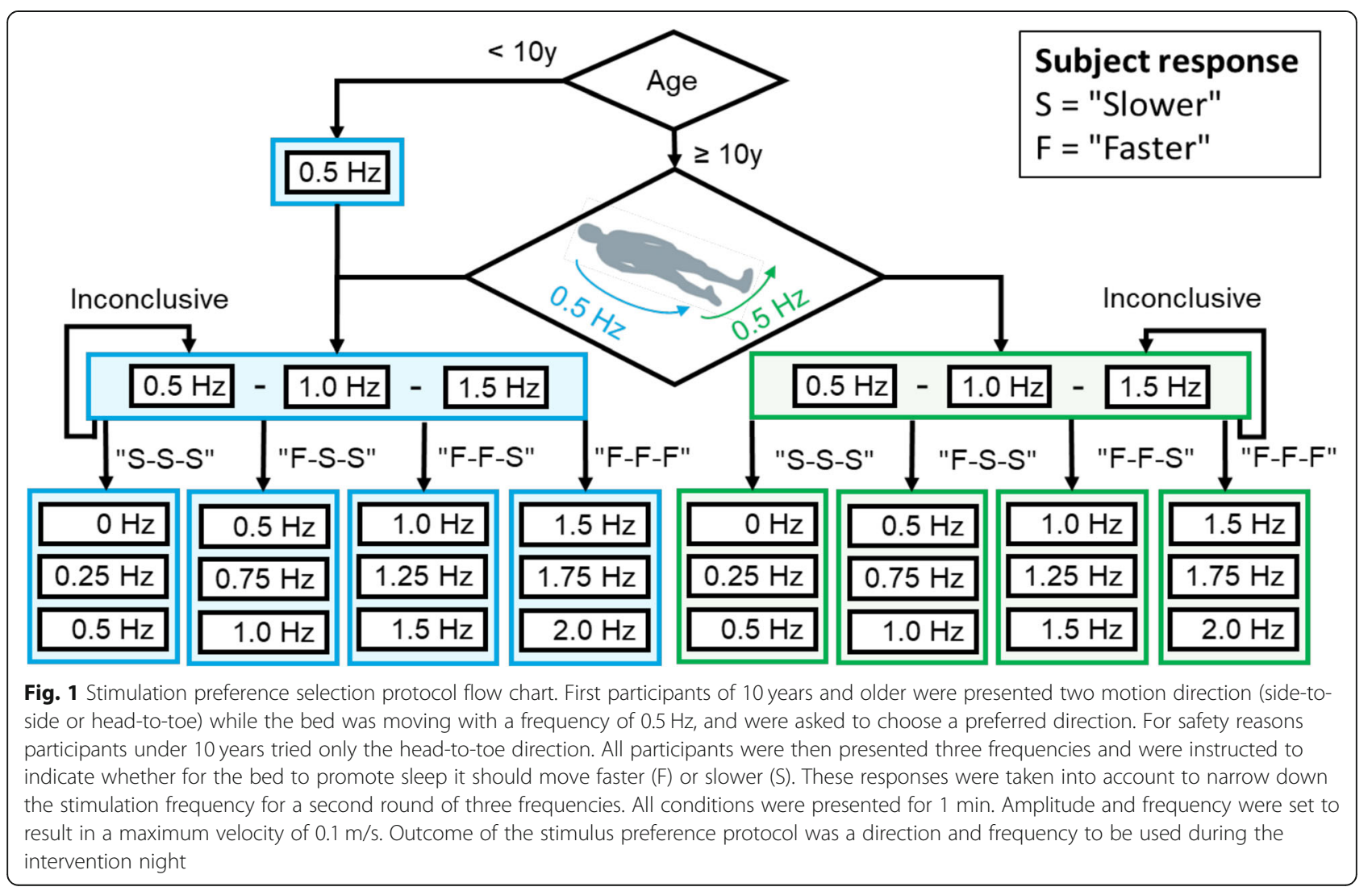


combination of settings, each for $1 \mathrm{~min}$, with participants lying in their preferred sleeping position (Fig. 1). Participants rated comfort of the bed, and how sleepy they felt after each bout of stimulation using a customdesigned five-point visual rating scales $(5=$ maximum comfort and sleepiness) (Supporting Information Document 4). For children over 10 years, both movement directions (head-to-toe and side-to-side) were presented in a random order at a frequency of $0.5 \mathrm{~Hz}$. For children under 10 years of age only head-to-toe was presented for safety reasons. Preferred movement direction was then used to assess preferred the preferred frequency. Three frequencies $(0.5,1.0$ and $1.5 \mathrm{~Hz})$ were presented in random order and participants indicated whether the bed should move faster or slower to promote sleep. Depending on their responses, further frequencies were presented in increments of $0.25 \mathrm{~Hz}$ to narrow down the preferred stimulation setting.

\section{Night protocol}

Following a sleep laboratory acclimatisation night (normal bed), participants spent the intervention and baseline night (order randomised) in the Somnomat. Rocking movements provided by the Somnomat (intervention night) or a sound recording of the Somnomat (baseline night) were activated for $1 \mathrm{~h}$ after lights out and again upon detection of RMs in a live video stream (Supporting Information Documents 1) until 10-min after RMs had stopped. After the hour of movement at sleep onset the amount of consecutive stimulation was restricted to $30 \mathrm{~min}$ per episode and a total of $3 \mathrm{~h}$ per night. During the baseline night, a recording of the sound of the moving bed was played to the participants to control for the influence of auditory stimulation generated by the bed (Crivelli et al. 2014). The same criteria were used for activating the audio as were used for activating the bed. Participants were instructed not to consume caffeine in the $5 \mathrm{~h}$ prior to the agreed bedtime.

\section{Measurement and analysis}

Participants wore an actigraphy watch on the nondominant wrist, and parents completed a sleep diary, to assess whether sleep in the laboratory was representative of sleep at home during the week prior to the study (Basic Mini Motionlogger watch, Ambulatory Monitoring Inc., New York). The device was set to zero crossing mode and sleep duration (minutes) was calculated using the Sadeh algorithm validated for use with children (ActionW2 software) (Sadeh et al. 1994).

To check compliance with caffeine intake guideline, as well as sleepiness and comfort, questionnaires were filled out prior to lights off and upon awakening by the participant and their parent. In the sleep laboratory, we further recorded video-somnography in the form of whole night $2 \mathrm{D}$ video recordings, as well as $3 \mathrm{D}$ videos, which have been reported elsewhere (Gall et al. 2019).

Video recordings (2D) were visually analysed according to the following criteria: An episode of rhythmic movement was defined as three or more consecutive rhythmic movements. The end of an episode was defined as a pause longer than the duration of two movements. Movements were scored based on visual inspection by two researchers: once online during the experiment and once offline. Number and duration of episodes were calculated over the common length of baseline and intervention night of each participant.

The habitual movement frequency was determined by dividing the number of rhythmic movements of one episode by the duration of the episode. For one participant the habitual movement frequency was determined based on a voluntary demonstration of his rhythmic movements prior to his third evening in the laboratory, since no episodes were recorded at night.

\section{Statistics}

Descriptive statistics for all variables are reported as medians and interquartile ranges (IQR), due to the small sample size. Comfort and sleepiness ratings upon awakening, total time in bed, total duration of rhythmic movement, number of episodes and the average duration of episodes during the baseline and intervention night were compared numerically. Statistics was performed using IBM SPSS version 24.

\section{Results}

\section{Stimulation preference}

Participants chose frequencies both within their characteristic movement range $(0.5,1.0$ and $2.0 \mathrm{~Hz} ; n=3)$, as well as below $(0.25 \mathrm{~Hz} ; \mathrm{n}=3)$. The median habitual movement frequency was $1.1 \mathrm{~Hz}$ (IQR: 0.8 to $1.3 \mathrm{~Hz}$, Table 1) and this did not correlate with preferred Somnomat frequency (Fig. 2a). Median levels of reported comfort and sleepiness at the child's preferred bed frequency were 3.5 (IQR: 3 to 4.5 ) and 4 (IQR: 4 to 5) out of 5 respectively, with 5 indicating optimal comfort and maximal sleepiness (Fig. 2b).

Of the three participants that were older than 10 years and therefore eligible to choose a movement direction, two chose the side-to-side direction. The chosen Somnomat movement direction matched the habitual rhythmic movement direction for one participant, one chose a direction that was not in line with their habitual direction, and one participant had a habitual movement direction (head banging on pillow) not simulated by the Somnomat.

To assess reliability, the responses to two trials of the preference protocol during which the children were provided with the same direction and frequency were 

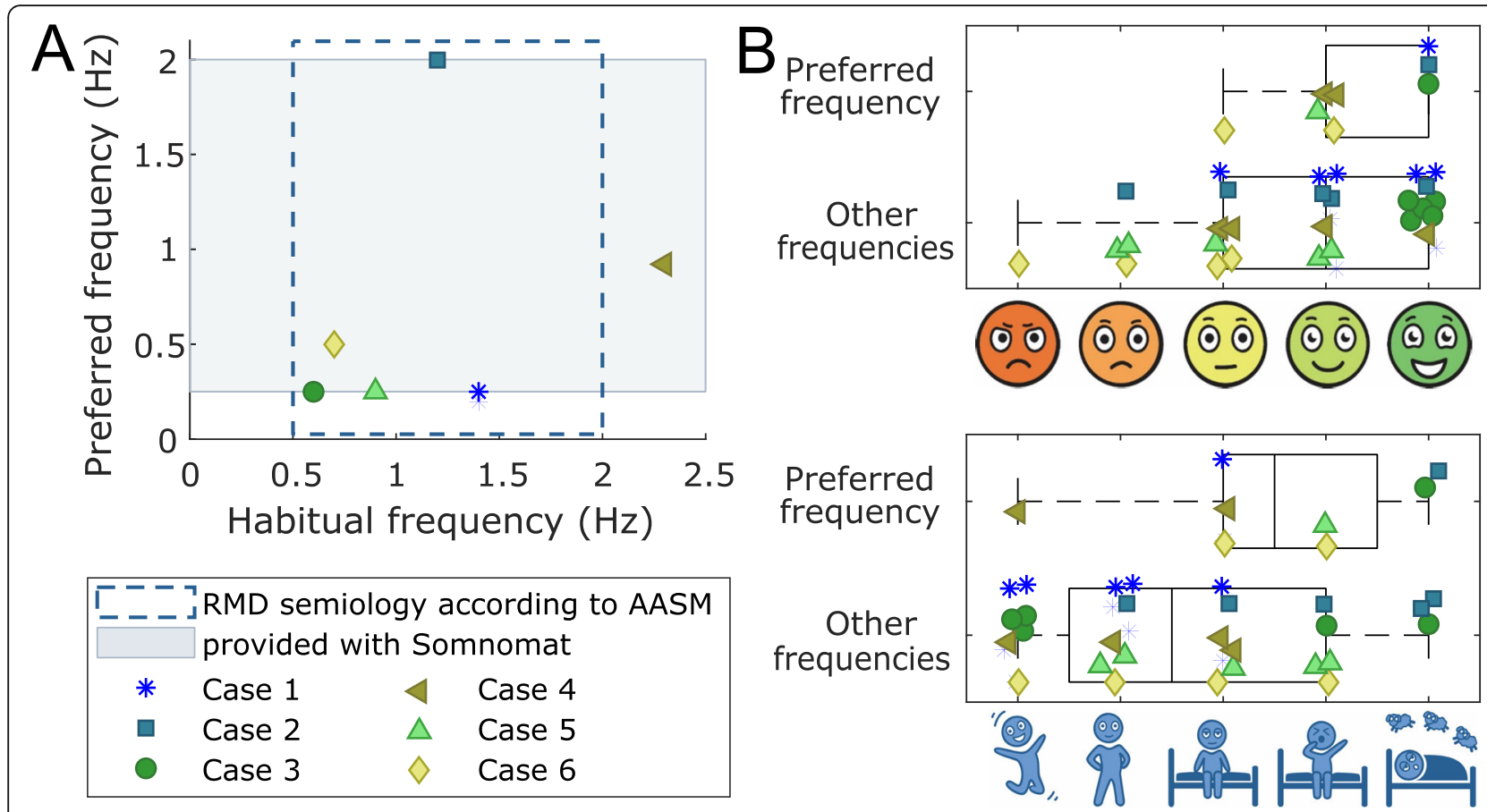

\begin{tabular}{|c|c|c|c|}
\hline I-- & $\begin{array}{l}\text { RMD se } \\
\text { provide }\end{array}$ & $\begin{array}{l}\text { gy } \\
\mathrm{Sc}\end{array}$ & $\begin{array}{l}\text { cording } \\
\text { inomat }\end{array}$ \\
\hline * & Case 1 & $\Delta$ & Case 4 \\
\hline$\square$ & Case 2 & $\triangle$ & Case 5 \\
\hline 0 & Case 3 & $\diamond$ & Case 6 \\
\hline
\end{tabular}

Other equencies

Fig. 2 Outcome of the movement preference protocol regarding frequency of the bed movement $(n=6)$. A. Habitual movement frequency while making rhythmic movements vs. the preferred bed frequency as determined during the preference protocol. Area indicated in blue shows the frequency range of rhythmic movement disorder (RMD) reported by the American Academy of Sleep Medicine (AASM). Area shaded grey shows the range of frequency provided using the Somnomat. B. Boxplots of comfort (top) and sleepiness (bottom) at the preferred bed frequency and all other frequencies combined

compared. Five out of six children responded consistently to the question 'For the bed to help you sleep should it move faster or slower?'. Comfort was rated more consistently $\left(\right.$ Median $_{\text {difference: }} 0, \mathrm{IQR}_{\text {difference: }} 0$ to 1) than sleepiness $\left(\right.$ Median $_{\text {difference: }}-1, \mathrm{IQR}_{\text {difference: - }}$ 1.5 to -0.5$)$.

\section{Performance of the rocking bed}

The accuracy of the stimulation, in the form of the root mean square error in velocity of the Somnomat, during rhythmic movements (Median: $0.0023 \mathrm{~m} / \mathrm{s}$; IQR: 0.0019 to $0.0092 \mathrm{~m} / \mathrm{s}$ ) was lower than while participants were lying still in the bed (Median: $0.0015 \mathrm{~m} / \mathrm{s}$; IQR: 0.0014 to $0.0028 \mathrm{~m} / \mathrm{s}$ ). In both cases, the error was minimal compared to the intervention maximum velocity $(0.1 \mathrm{~m} / \mathrm{s})$. For one participant with two movement semiologies, a larger velocity error in the movement direction of the bed was observed when rhythmic movement occurred in the direction of the bed $(0.0033 \mathrm{~m} / \mathrm{s})$ compared to when movement was perpendicular to the movement of the bed $(0.0015 \mathrm{~m} / \mathrm{s})$.

During one intervention night the variability induced by the moving participant led to a mismatch between the bed reference and target position, twice leading to a safety stop. At the frequency of $2.0 \mathrm{~Hz}$ (upper limit) the amplitude was $0.001 \mathrm{~m}$, a displacement very close to the resolution of the safety sensor used to monitor bed motion. By adapting the safety configuration to trajectories with small amplitudes this problem can be avoided. Researcher error resulted in the control intervention starting $12 \mathrm{~min}$ late on one occasion (Case 5) and ending 8 min late on another occasion (Case 4). No episodes of rhythmic movements were missed by the experimenters based on the comparison of the online scoring and offline scoring of the video data.

\section{Acceptability and self-reported feedback on night protocol}

All children reported feeling safe in the Somnomat and could imagine having a rocking bed at home. Parents also reported feeling their child was safe and five of six parents could imagine having the rocking bed at home. Four children thought the bed could help their RMD, the other two were unsure. After trying out the bed overnight, four out of six children thought it could help them sleep, one was unsure and one did not think it would be helpful.

The children reported feeling more rested upon awakening after the intervention night (Median: 1.5, IQR: 1 to 2) than after the baseline night (Median: 2, IQR: 1.25 to 2.75). Participants also felt more comfortable upon awakening after the intervention night (Median: 4.5, 
IQR: 4 to 5) than after the baseline night (Median: 4, IQR: 3.25 to 4.75$)$.

Three participants (case 2, 3 and 5) rated their time to fall asleep more positively during intervention nights than during baseline nights. However, one participant thought they woke up more frequently (case 1) and one felt a stronger urge to move (case 1).

\section{Effect of bed movement on symptoms}

Sleep duration based on actigraphy was similar during the baseline night (Median: $503 \mathrm{~min}, \mathrm{IQR}: 446$ to 532 min, $n=3$ ) compared to the average of the nights at home (Median: $477 \mathrm{~min}$, IQR: 446 to $497 \mathrm{~min}$ ). Based on videosomnography analysis the children spent between 0 and $40 \%$ of the time in bed engaged in rhythmic movements. The duration of rhythmic movement did not differ between baseline (Median: 3.3\%, IQR: 0.4 to $17.1 \%$ ) and intervention nights (Median: 3.2\%, IQR: 0.7 to 17.9\%). Neither did time in bed, number of episodes and the average duration of episodes (Fig. 3).

Case 5 showed symptoms during the baseline, but not during the intervention night (preferred movement in habitual movement direction). Case 2 was able to turn 90 degrees in the bed and was stimulated in his habitual movement direction for $27 \mathrm{~min}$ and perpendicular to his habitual movement direction for $130 \mathrm{~min}$. When stimulated in his habitual movement direction, episodes were shorter and less frequent (5.11 episodes/hour; Medianduration: $0.80 \mathrm{~min}$; IQR duration: 0.73 to $1.83 \mathrm{~min}$ ) compared to other directions (17.6 episodes/hour; Median duration: $1.56 \mathrm{~min}$; IQR duration: 0.63 to $2.04 \mathrm{~min}$ ). Case 4 did not show symptoms on either night. For the other three participants no effect of bed movement was observed (Fig. 4).

\section{Discussion}

The primary aim of this study was to test the feasibility and acceptability of rhythmic vestibular stimulation as therapy for sleep-related rhythmic movement disorder, which could be shown based on device performance and participant feedback. With our small sample, measured during one night in the laboratory, no significant clinical outcomes were expected. Indeed, unlike the case series reporting on use of hammocks in the home environment (Hewertson and Hill 2018), we could not show a positive effect on rhythmic movements using a rocking bed for one night in the laboratory. For all five reported cases a resolution of rhythmic movements whilst using the hammock in the home environment was observed (Hewertson and Hill 2018). Timing and intensity of hammock use was not recorded, but the hammock was available for use on a daily basis and over a longer period, which might be essential for clinical efficacy. One of the five children who returned to a normal bed after 5 weeks of hammock use did not relapse, suggesting that effects of sensory stimulation might have lasting effects.

A limitation of the current study was that the available movement directions of the bed were limited to head-totoe and side-to-side and, therefore, did not include the habitual directions of movement of all children. Furthermore, for safety reasons, participants under 10 years of age were exposed to only head-to-toe movement. This could explain why the movement direction and frequency preferred by children did not reliably match that of their habitual rhythmic movements. Moreover, for one participant who moved 90 degrees in his bed during the night, the bed movement direction seemed to effect treatment outcome. When stimulated in the direction of habitual movement, the episodes of rhythmic movement were shorter and less frequent compared to movements perpendicular to his habitual movement direction. It has been suggested that the frequency of vestibular stimulation modulates the influence of the stimulation on sleep (Vrugt and Pederson 1973). For future trials it might be of interest to see if emulating the direction and frequency of habitual rhythmic movements or rather

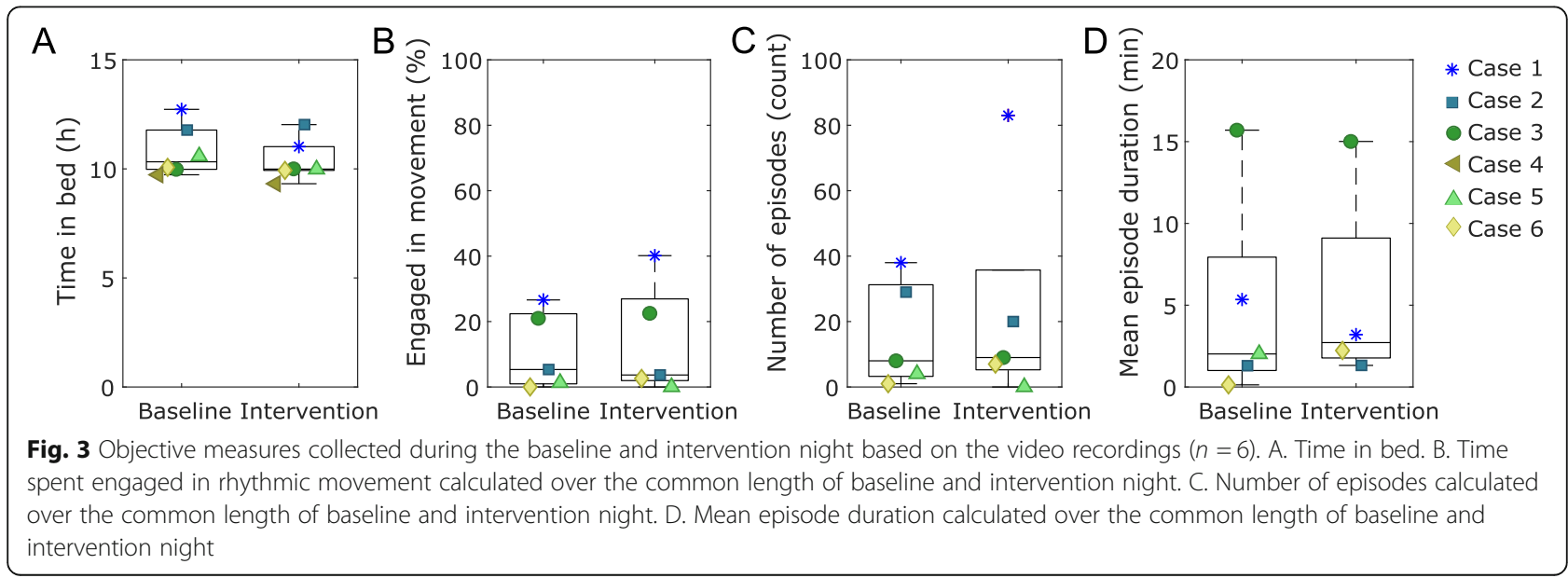




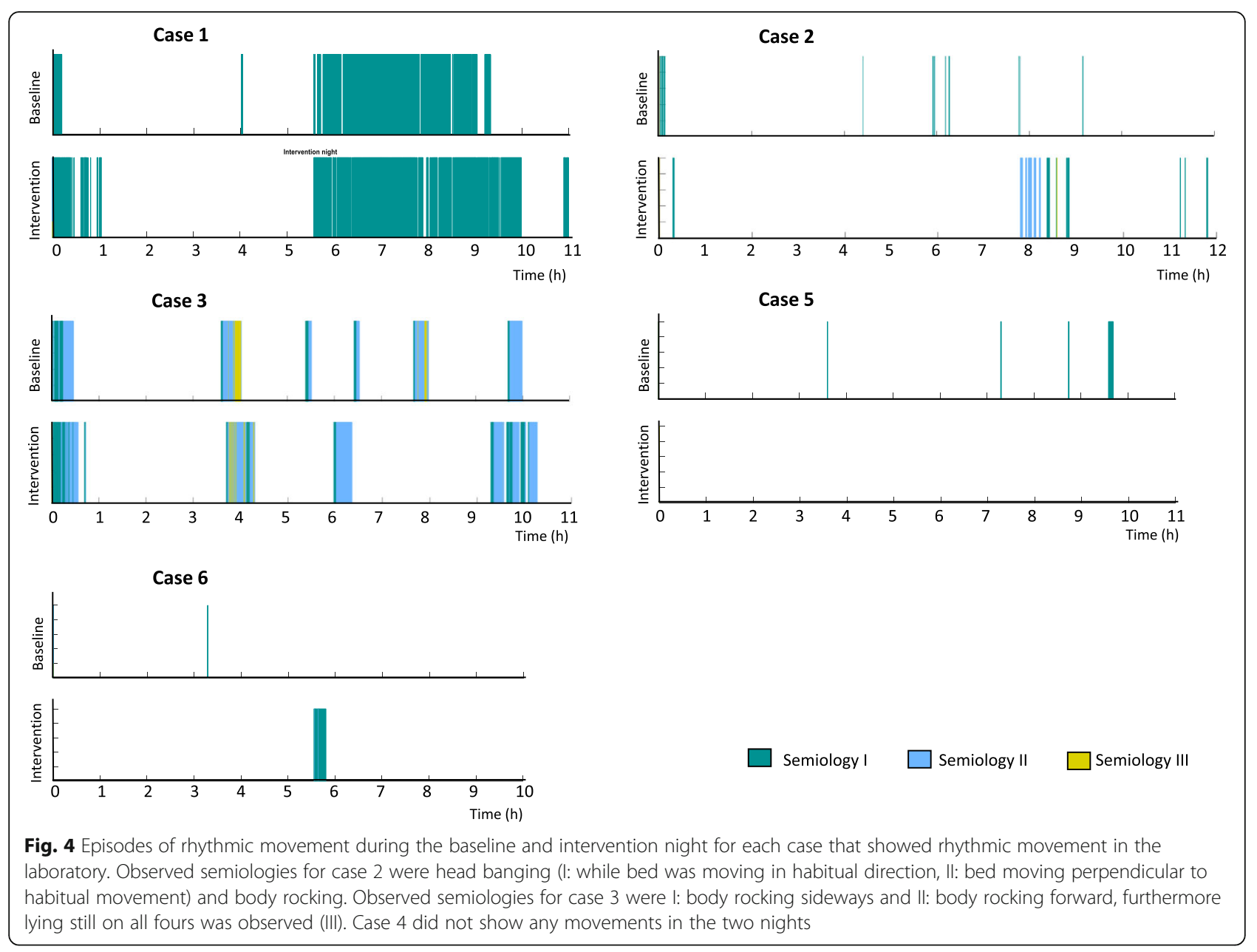

finding the most comfortable setting reported by the participants, as was done in this study, has higher therapeutic value. If the first is true, than it seems the vestibular stimulation generated by the patient's movements is effectively inducing sleep, where if the latter is true it might be likely that the patient's movements are a selfsoothing behaviour.

A challenge when studying RMD is that not all patients engage in rhythmic movement during polysomnographic recordings in a laboratory setting (Stepanova et al. 2005). This is confirmed by our clinical experience. In this study, with only contactless video-somnography recording, five out of six participants engaged in rhythmic movements in the laboratory setting. Solely one child (case 4), who was included in the study based on video evidence of rocking in the home environment, did not rock in the sleep laboratory. To increase the proportion of participants that engage in rhythmic movement in an experimental setting, moving the treatment and measurements to the home environment is recommended.

We see significant within child night-to-night variability of both number and duration of episodes of rhythmic movements during clinical home video recordings. Thus, to study the efficacy of our proposed therapy, evaluation over a longer exposure period and in the home setting would be ideal. Domiciliary rocking therapy would require an automated system. Two previous studies reported on acoustically based online applications potentially suited to RMD therapy, in one case supplemented by body worn sensors. In the present study the intervention was controlled by experimenters continually observing a 2D video stream. Contactless technologies, such as radar and 3D video, have the potential to automatically detect abnormal movements during sleep (Rahman et al. 2015; Garn et al. 2016). For future studies a closed loop system based on movement-sensitive acoustic sensors, radar or 3D video, would allow this promising new therapy to be studied in the ecologically relevant home setting.

\section{Conclusion}

Application of rocking movements in children with rhythmic movement disorder using the Somnomat was technically feasible. The sometimes violent movements 
generated by the participants only minimally perturbed the movement of the bed, resulting in reliable execution of the planned bed movement. After trying out the bed, children reported the bed movements to be comfortable. Furthermore, children were more comfortable and were less sleepy upon awakening after the intervention night than after the baseline night. All children and their parents perceived the bed to be safe and the majority of children and parents believed the bed could help reduce rhythmic movements and promote sleep.

Considering the limited scientific evidence on therapies for sleep-related rhythmic movement disorder, a theoretically driven potential therapy substituting patient rhythmic movements with an external source of sensory stimulation, deserves further evaluation.

\section{Supplementary information}

Supplementary information accompanies this paper at https://doi.org/10. 1186/s41606-020-00049-9.

Additional file 1. Supplemental information document 1. Video showing the start of an episode of rhythmic movement.

Additional file 2. Supplemental information document 2. Video of an episode of rhythmic movement in a patient with two movement semiologies. The patient switches from one semiology (side-to-side rocking) to another semiology (forward rolling movement) without pause. Often this occurs multiple times in an episode of rhythmic movement.

Additional file 3. Supplemental information document 3. The Rhythmic Movement Disorder Questionnaire. A structured interview for diagnosis of sleep-related rhythmic movement disorder in children

Additional file 4. Supplemental information document 4. Pediatric visual rating scales for comfort and sleepiness.

\section{Abbreviations}

IQR: Interquartile range; RMD: Rhythmic movement disorder

\section{Acknowledgements}

We would like to thank the Facebook group 'Rhythmic movement support' for assisting recruitment and all parents of participants for their effort without which this study could not have been conducted. We are grateful to Michael Herold-Nadig, and David Renggli for their help in adapting the setup to the paediatric population, and to Marina Schnauss and Vanessa Hewertson for their support during data collection. A special thank you goes to Julia van Sluijs for design of the paediatric visual rating scales for comfort and sleepiness.

\section{Authors' contributions}

The study was designed by RS, EW, L, OJ, and $\mathrm{CH}$. Device and sensor set-up were developed by RS, EW, QR, MG, PA, and RR. Data was collected by RS, EW, $\mathrm{QR}$, and $\mathrm{CH}$. Data processing and analysis was performed by RS, EW, MG, and PA. The manuscript was written by RS and $\mathrm{CH}$. The manuscript was critically reviewed by all co-authors. The author(s) read and approved the final manuscript.

\section{Funding}

This study was funded by the Southampton University Global Partnership fund, the Swiss National Science Foundation (Grant CR3213-162809/1) and the Commission for Technology and Innovation (Grant 17988). The funding bodies did not influence the design of the study and collection, analysis, and interpretation of data or writing of the manuscript.

\section{Availability of data and materials}

The video data generated and/or analysed during the current study are not publicly available, because this data could not be anonymized. The manual scoring of the video data, supporting the conclusions of this article, is available in the ETH research collection repository: DOI https://doi.org/10 3929/ethz-b-000392724, http://hdl.handle.net/20.500.11850/392724.

\section{Ethics approval and consent to participate}

The UK National Research Ethics Committee and Health Research Authority (IRAS 234505), the Cantonal Ethics Committee of Zurich (KEK 2017-01880) and Swissmedic (2017-MD-0035) approved the study. All children gave informed assent and the parent gave informed consent.

\section{Consent for publication}

The parents of the participant visible in the supplemental video signed a consent form for clinical photography, stating that the video material can be used for presentation at scientific meetings, teaching purposes and publication in a scientific journal.

\section{Competing interests}

Authors report no conflicts of interest.

\section{Author details}

${ }^{1}$ Department of Health Sciences and Technology, Sensory-Motor Systems Lab, ETH Zurich, Sonneggstrasse 3, 8092 Zurich, Switzerland. ${ }^{2}$ Center of Competence Sleep \& Health Zurich, University of Zurich, Frauenklinikstrassse 28, 8091 Zurich, Switzerland. ${ }^{3}$ Austrian Institute of Technology, Giefinggasse 4, 1210 Vienna, Austria. ${ }^{4}$ Institute for Pharmacology and Toxicology, University of Zurich, Winterthurerstrasse 190, 8057 Zurich, Switzerland. ${ }^{5}$ Child Development Center, University Children's Hospital Zurich, Steinwiesstrasse 75, 8032 Zurich, Switzerland. ${ }^{6}$ Medical Faculty, University of Zurich, Forchstrasse 340, 8008 Zurich, Switzerland. ${ }^{7}$ Childrens Sleep Disorder Service, Southampton Children's Hospital, Tremona Road, Southampton SO16 6YD,

UK. ${ }^{8}$ School of Clinical and Experimental Sciences, Faculty of Medicine,

University of Southampton, Tremona Road, Southampton SO16 6YD, UK.

Received: 24 January 2020 Accepted: 8 July 2020

Published online: 29 July 2020

\section{References}

Barnard KE, Bee HL. The impact of temporally patterned stimulation on the development of preterm infants. Child Dev. 1983;54(5):1156-67.

Bayer L, Constantinescu I, Perrig S, Vienne J, Vidal P-P, Mühlethaler M, et al. Rocking synchronizes brain waves during a short nap. Curr Biol. 2011;21(12): $461-2$

Clark DL, Chee F. Vestibular stimulation influence on motor development in infants. Science. 1977;196(4295):1228-9.

Crivelli F, Heinicke L, Omlin X, Riener R, Somnomat: A novel device to investigate the influence of vestibular stimulation on sleep. 5th IEEE RAS/EMBS International Conference on Biomedical Robotics and Biomechatronics; 2014: IEEE.

Crivelli F, Omlin X, Rauter G, Von Zitzewitz J, Achermann P, Riener R. Somnomat: a novel actuated bed to investigate the effect of vestibular stimulation. Med Biol Eng Comput. 2016;54(6):877-89.

Gall M, Kohn B, Wiesmeyr C, Van Sluijs RM, Wilhelm E, Rondei QJ, et al. A novel approach to assess sleep-related rhythmic movement disorder using automatic 3D analysis. Front Psychiatry. 2019;10:709.

Garn H, Kohn B, Dittrich K, Wiesmeyr C, Kloesch G, Stepansky R, et al., 3D detection of periodic limb movements in sleep. 2016 IEEE 38th Annual International Conference of the Engineering in Medicine and Biology Society (EMBC): 2016: IEEE.

Gogo E, van Sluijs RM, Cheung T, Gaskell C, Jones L, Alwan NA, et al. Objectively confirmed prevalence of sleep-related rhythmic movement disorder in preschool children. Sleep Med. 2018;53:16-21.

Golbin A, Guseva V, Shepovalnikov A. Unusual behaviors in sleep as "compensatory" reactions, aimed at normalizing the sleep-wake cycle. Hum Physiol. 2013;39(6):635-41

Grabherr L, Macauda G, Lenggenhager B. The moving history of vestibular stimulation as a therapeutic intervention. Multisens Res. 2015;28(5-6):653-87.

Gwyther A, Walters A, Hill C. Rhythmic movement disorder in childhood: an integrative review. Sleep Med Rev. 2017;35:62-75. 
Haywood PM, Hill CM. Rhythmic movement disorder: managing the child who head-bangs to get to sleep. Paediatrics Child Health. 2012;22(5):207-10.

Henriques IF, De Oliveira DWD, Oliveira-Ferreira F, Andrade PM. Motion sickness prevalence in school children. Eur J Pediatr. 2014;173(11):1473-82.

Hewertson V, Hill CM. Rhythmic movement disorder: a novel solution? \#P58, 5th International Pediatric Sleep Association; 2018.

Mahowald M. Non-arousal parasomnias in the child. Principles practice of sleep medicine in the child; 1995. p. 115-23.

Manni R, Terzaghi M. Rhythmic movements during sleep: a physiological and pathological profile. Neurol Sci. 2005;26(3):s181-s5.

Mindell JA, Owens JA. A clinical guide to pediatric sleep: diagnosis and management of sleep problems: Lippincott Williams \& Wilkins; 2015.

Omlin X, Crivelli F, Heinicke L, Zaunseder S, Achermann P, Riener RJM. Effect of rocking movements on respiration. PLoS One. 2016;11(3):e0150581.

Rahman T, Adams AT, Ravichandran RV, Zhang M, Patel SN, Kientz JA, et al, Dopplesleep: A contactless unobtrusive sleep sensing system using shortrange doppler radar. Proceedings of the 2015 ACM International Joint Conference on Pervasive and Ubiquitous Computing; 2015: ACM.

Sadeh A, Sharkey M, Carskadon MA. Activity-based sleep-wake identification: an empirical test of methodological issues. Sleep. 1994;17(3):201-7.

Sallustro F, Atwell CW. Body rocking, head banging, and head rolling in normal children. J Pediatr. 1978:93(4):704-8.

Sateia MJ. International classification of sleep disorders; 2014. p. 1387-94.

Shibagaki H, Ashida K, Morita Y, Ikeura R, Yokoyama K. Verifying the sleepinducing effect of a Mother's rocking motion in adults. J Robotics Networking Artificial Life. 2017:4(2):129-33.

Shneerson JM. Sleep medicine: a guide to sleep and its disorders: John Wiley \& Sons; 2009 .

Stepanova I, Nevsimalova S, Hanusova J. Rhythmic movement disorder in sleep persisting into childhood and adulthood. Sleep. 2005;28(7):851-7.

Vrugt DT, Pederson DR. The effects of vertical rocking frequencies on the arousal level in two-month-old infants. Child Dev. 1973;44(1):205-9.

\section{Publisher's Note}

Springer Nature remains neutral with regard to jurisdictional claims in published maps and institutional affiliations.

Ready to submit your research? Choose BMC and benefit from:

- fast, convenient online submission

- thorough peer review by experienced researchers in your field

- rapid publication on acceptance

- support for research data, including large and complex data types

- gold Open Access which fosters wider collaboration and increased citations

- maximum visibility for your research: over $100 \mathrm{M}$ website views per year

At $\mathrm{BMC}$, research is always in progress.

Learn more biomedcentral.com/submissions 